\title{
Age of Rats Affects the Degree of Retinal Neuroinflammatory Response Induced by High Acute Intraocular Pressure
}

\author{
Shuhan Meng $\mathbb{D},{ }^{1,2,3}$ Dan Wen $\mathbb{D}^{1,2,3}$ Jingge Xiao ${ }^{10},{ }^{4}$ Qianyue Zhang $\left(\mathbb{D},{ }^{4}\right.$ \\ Weizhou Fang ${ }^{1 D},{ }^{1,2,3}$ Xiao Xue ${ }^{4},{ }^{4} \mathrm{Tu} H u\left(D,{ }^{1,2,3}\right.$ and Xiaobo Xia ${ }^{1,2,3}$ \\ ${ }^{1}$ Eye Center of Xiangya Hospital, Central South University, Changsha, Hunan, China 410008 \\ ${ }^{2}$ Hunan Key Laboratory of Ophthalmology, Changsha, Hunan, China 410008 \\ ${ }^{3}$ National Clinical Research Center for Geriatric Disorders, Xiangya Hospital, Central South University, Changsha, Hunan, China \\ 410008 \\ ${ }^{4}$ Xiangya Medical School, Central South University, Changsha, Hunan, China 410013
}

Correspondence should be addressed to Tu Hu; hutu1986@csu.edu.cn and Xiaobo Xia; xbxia21@csu.edu.cn

Received 27 November 2021; Accepted 8 January 2022; Published 29 January 2022

Academic Editor: Liu Jinhui

Copyright ( 2022 Shuhan Meng et al. This is an open access article distributed under the Creative Commons Attribution License, which permits unrestricted use, distribution, and reproduction in any medium, provided the original work is properly cited.

Purpose. To investigate whether retinal neuroinflammatory response was affected by aging in a rat model of acute glaucoma. Methods. Young adult and aged rats were randomly assigned into normal control, $45 \mathrm{mmHg}, 60 \mathrm{mmHg}$, and $90 \mathrm{mmHg}$ groups. Intraocular pressure (IOP) of rats was acutely elevated to $45 \mathrm{mmHg}, 60 \mathrm{mmHg}$, and $90 \mathrm{mmHg}$, respectively. Three days after high IOP treatment, loss of retinal ganglion cells (RGCs), formation of proinflammatory microglia/macrophages and neurotoxic astrocytes, and deposition of complement $\mathrm{C} 3$ in the retina were detected by immunofluorescence. ELISA was used to assess the protein levels of proinflammatory cytokines TNF and IL- $1 \beta$ in the retina. Results. Compared with young adult retinae, (1) loss of RGCs was more severe in aged retinae under the same IOP treatment, (2) microglia/macrophages were more prone to adopt proinflammatory phenotype in aged retinae in response to elevated IOP, (3) high IOP treatment induced astrogliosis, formation of neurotoxic astrocytes, and deposition of complement C3 more easily in aged retinae, and (4) aged retinae induced higher levels of proinflammatory cytokines TNF and IL- $1 \beta$ under the same IOP treatment. Conclusion. Our data indicated that aging affects the degree of retinal neuroinflammatory response initiated by ocular hypertension, which may contribute to the age-related susceptibility of RGCs to elevated IOP.

\section{Introduction}

Glaucoma is an age-related neurodegenerative disease and the leading cause of irreversible blindness worldwide [1]. It is marked by the degeneration of retinal ganglion cell (RGC) axons, soma, and synapses [2]. Clinical studies have clearly established that increased intraocular pressure (IOP) is the major risk factor in glaucoma [3]. By now, therapeutic strategy for all types of glaucoma is limited to the reduction of elevated IOP, which does not completely stop the progression of glaucomatous neurodegeneration and visual field defects [4]. Besides IOP, age is another main risk factor of glaucoma [5, 6]. A body of studies has demonstrated that the prevalence of glaucoma increases markedly with advancing age across all populations $[1,7,8]$.
Consistent with these findings, we previously identified the existence of the age-related susceptibility of rat retinae to increased IOP [9]. Other reports suggested that agerelated mitochondria dysfunction and accumulation of oxidative damage make RGCs more vulnerable to damage in the progression of glaucomatous neurodegeneration [10, 11]. Moreover, chronic exposure of tissue stress (oxidized lipoproteins and free radicals) triggered the low-grade inflammation (parainflammation) in aged retinae [12]. A physiological level of parainflammation was critical for maintaining the retinal homeostasis, but when injuries occurred, dysregulated parainflammation developed into detrimental inflammatory response which exacerbates the glaucomatous neurodegeneration [13]. Similar to other neurodegenerative diseases, resident microglia and astrocytes as 
well as infiltrated macrophages participated in the retinal neuroinflammatory response in glaucoma [14]. These immune cells could recognize and respond to various stimuli including glaucoma-related stress and damage-associated molecular patterns (DAMPs) arising from stressed or damaged RGCs [15]. Recent studies have well established that microglia/macrophages and astrocytes can be activated by these stimuli and transform into the proinflammatory/neurotoxic phenotype [16-19]. In glaucoma, these proinflammatory microglia/macrophages and neurotoxic astrocytes produced proinflammatory cytokines (e.g., tumor necrosis factor (TNF), interleukin-1 $\beta$ (IL-1 $\beta$ ), IL-6, and IL-12), chemokines (e.g., C-C motif chemokine ligand 2 (CCL2), C-XC motif chemokine ligand 2 (CXCL2), and CXCL10), complement component (e.g., C1q, C3, and C4), reactive oxygen species (ROS), and nitric oxide (NO) to directly induce RGC death or amplify the retinal neuroinflammatory response [16].

Recent evidence hinted that some microglia and astrocytes lose their ability to maintain homeostasis and adopt a more proinflammatory or "primed" phenotype as a result of the aging process [20-22]. Compared to the resting microglia and astrocytes, these "primed" microglia and astrocytes were more sensitive to inflammatory cues and displayed the more robust inflammatory response, as the higher expression of proinflammatory cytokines/chemokines and complement component $[23,24]$. Our previous results showed that high IOP treatment induces the more evident activation of microglia/macrophages (characterized by the more IBA1- (ionized calcium-binding adapter molecule 1-) positive cells and IBA1-positive stained area) in aged retinae than young adult retinae [9]. However, the proinflammatory features of microglia/macrophages have not been identified in this scenario.

In the present study, we detected the formation of proinflammatory microglia/macrophages and neurotoxic astrocytes, deposition of complement $\mathrm{C} 3$, and production of proinflammatory cytokines (TNF and IL-1 $\beta$ ) in the rat glaucoma model we previously used [9]. These findings raised the possibility that retinal neuroinflammatory response may contribute to the age-related vulnerability of RGCs in glaucoma.

\section{Materials and Methods}

2.1. Animal and Grouping. Thirty-six young adult (aged 2 months, 200-250 g) and thirty-six aged (aged 15 months, 300-350 g) female Sprague-Dawley rats were supplied from Central South University, Changsha, Hunan Province, People's Republic of China. All rats were housed under controlled environmental conditions on a $12 \mathrm{~h}$ light/dark cycle with ad libitum access to food and water. All experiments were approved by the animal ethics committee in the Xiangya Hospital of Central South University. Young adult and aged rats were randomly divided into normal control $(n=9 ; n=3,6$ eyes for retinal whole mounts; $n=3,6$ eyes for retinal cross-sections; and $n=3,6$ eyes for ELISA test), $45 \mathrm{mmHg}(n=9 ; n=3,6$ eyes for retinal whole mounts; $n$ $=3,6$ eyes for retinal cross-sections; and $n=3,6$ eyes for
ELISA test), $60 \mathrm{mmHg}(n=9 ; n=3,6$ eyes for retinal whole mounts; $n=3,6$ eyes for retinal cross-sections; and $n=3,6$ eyes for ELISA test), and $90 \mathrm{mmHg}(n=9 ; n=3,6$ eyes for retinal whole mounts; $n=3,6$ eyes for retinal crosssections; and $n=3,6$ eyes for ELISA test) groups. IOP of rats in $45 \mathrm{mmHg}, 60 \mathrm{mmHg}$, and $90 \mathrm{mmHg}$ groups was increased acutely to $45 \mathrm{mmHg}, 60 \mathrm{mmHg}$, and $90 \mathrm{mmHg}$, respectively. All rats were sacrificed with an i.p. overdose of pentobarbital at 3 days after high IOP treatment.

2.2. Acute IOP Model. The animal model was prepared following the reported method $[9,25]$. Briefly, under anesthesia of $2 \%$ pentobarbital $(40 \mathrm{mg} / \mathrm{kg})$, a 30 -gauge infusion needle connected to the installation instrument filled with sterile saline was inserted into the anterior chamber of rats. The IOP was raised to $45 \mathrm{mmHg}, 60 \mathrm{mmHg}$, or $90 \mathrm{mmHg}$ for $60 \mathrm{~min}$. After maintenance of $60 \mathrm{~min}$, the $90 \mathrm{mmHg}$ of IOP was decreased through $80 \mathrm{mmHg}$ for $5 \mathrm{~min}, 70 \mathrm{mmHg}$ for $5 \mathrm{~min}, 60 \mathrm{mmHg}$ for $5 \mathrm{~min}$, and $30 \mathrm{mmHg}$ for $5 \mathrm{~min}$. For the condition of $45 \mathrm{mmHg}$ and $60 \mathrm{mmHg}$ of IOP, the IOP was directly decreased to $30 \mathrm{mmHg}$ after maintenance of $60 \mathrm{~min}$.

2.3. Retinal Tissue Preparation. After anesthetizing, rats were transcardially perfused with ice-cold $0.9 \%$ saline followed by ice-cold $4 \%$ paraformaldehyde. The eyeballs were enucleated, and the corneas, lenses, and vitreous bodies were removed. For retinal cross-sections, the remaining eye cups were postfixed overnight in $4 \%$ paraformaldehyde at $4^{\circ} \mathrm{C}$, placed into $30 \%$ sucrose for cryoprotection, and embedded in OCT compound (Tissue-Tek). The $20 \mu \mathrm{m}$ tissue sections were prepared with a Leica cryostat. For retinal whole mounts, the remaining eye cups were postfixed in $4 \%$ paraformaldehyde for $1 \mathrm{~h}$, and the whole retinas were dissected and processed as described previously [26].

For ELISA, rats were transcardiac perfused with ice-cold $0.9 \%$ saline. The eyeballs were enucleated and retinae were collected. Then retinae were homogenized by sonication on ice in a digestion buffer containing protease inhibitors (Sigma). The homogenates were treated with centrifugation, protein concentration determination, and denaturation.

2.4. Immunofluorescence. Retinal whole mounts and crosssections were blocked with PBS containing 5\% BSA and $0.3 \%$ Triton X-100 for $1 \mathrm{~h}$ at room temperature and incubated with primary antibodies diluted in PBS containing $5 \%$ BSA and $0.1 \%$ Triton X-100 overnight at $4^{\circ} \mathrm{C}$ (rabbit anti-rat RBPMS (RNA-binding protein with multiple splicing), GeneTex, GTX118619, 1:500; rabbit anti-rat IBA1, Wako, 019-19741, 1:200; mouse anti-rat CD68, Abcam, ab31630, 1:200; mouse anti-rat MHC-II (major histocompatibility complex class II), Abcam, ab23990, 1:200; mouse anti-rat GFAP (glial fibrillary acidic protein), Cell Signaling Technology, \#3670, 1:2000; and rabbit anti-rat C3, Abcam, ab200999, 1:200). Then the retinal whole mounts and cross-sections were incubated with secondary antibodies labeled with fluorescent dyes $(1: 1000$, Jackson ImmunoResearch) for $2 \mathrm{~h}$ at room temperature followed by nuclei staining with DAPI (Vector Laboratories). As negative controls, 
an adjacent series of sections were processed in parallel without the primary antibodies.

2.5. Enzyme-Linked Immunosorbent Assay Analysis (ELISA). The levels of secreted TNF and IL- $\beta$ in the retinal supernatants were detected by rat TNF (\#CSB-E11987r, CUSABIO, China) and IL-1 $\beta$ (\#CSB-E08055r, CUSABIO, China) ELISA kits. Briefly, the testing sample and standard sample were added in duplicates to plates precoated with the primary antibody and incubated for $2 \mathrm{~h}$ at $37^{\circ} \mathrm{C}$. Next, the biotinlabeled secondary antibody was added and incubated for $1 \mathrm{~h}$ at $37^{\circ} \mathrm{C}$. After incubation, HRP-conjugate reagent was added followed by repeat incubation. Then, tetramethylbenzidine enzyme-substrate (TMB) was added and incubated for $30 \mathrm{~min}$ at $37^{\circ} \mathrm{C}$. Finally, stop solution was added for stopping the reaction, and the absorbance (OD450) of all BSA Standards and samples were recorded within $5 \mathrm{~min}$.

2.6. Cell Counting and Data Analysis. The central and peripheral regions of the rat retina were defined as previously described [27]. For each retinal whole mount, twelve images from the central and peripheral region of the retina were taken under 20x magnification, respectively, using the Leica DM6000 fluorescence microscope. RBPMS-labeled RGCs in each $20 x$ field were counted. A total $\mathrm{RBPMS}^{+}$cell number of 12 fields per region were averaged to represent RGC density in the central and peripheral retinae, respectively.

Three sections from each eye for CD68/IBA1, MHC-II/ IBA1, and GFAP/C3 double staining were chosen. The proinflammatory microglia/macrophages were defined by colabeling of IBA1 (microglia/macrophages marker) and CD68 (phagocytic marker)/MHC-II (antigen presenting molecular). The neurotoxic astrocytes were defined by colabeling of GFAP (astrocyte marker) and complement C3. Two images from the central region for each retinal crosssection were randomly captured under 40x magnification using the Zeiss LSM780 confocal microscope. The number of $\mathrm{CD}^{+} 8^{+} \mathrm{IBA}^{+}$and $\mathrm{MHC}-\mathrm{II}^{+} \mathrm{IBA}^{+}$cells in captured images of the inner retina (including nerve fiber layer, ganglion cell layer, and inner plexiform layer) was blindly counted, respectively. Relative mean gray value and relative positive area of GFAP and C3 staining in the inner retina were measured by ImageJ.

All data were presented as mean \pm standard deviation (mean $\pm \mathrm{SD}$ ). Two-way analysis of variance (ANOVA) followed by Tukey's multiple comparisons test was used to assess the statistical significance. Assessments with $p<0.05$ were considered significant.

\section{Results}

3.1. The Same High IOP Caused More Serious Loss of RGCs in Aged Retinae than in Young Adult Retinae. Loss of RGCs is the major pathological hallmark of glaucoma [27]. Here, we assessed the RGC loss by counting the number of RBPMS- (a specific marker of RGC in the retina) labeled cells in retinal whole mounts of aged and young adult rats 3 days after IOP treatment of $45 \mathrm{mmHg}, 60 \mathrm{mmHg}$, and
$90 \mathrm{mmHg}$ (Figure 1). Two-way ANOVA analysis showed that aging exacerbates the loss of RGCs after IOP treatment $(F(1,40)=198.97, p<0.0001$, for central; $F(1,40)=110.26$, $p<0.001$, for peripheral). Moreover, increased IOP also exerted effects on RGC loss $(F(3,40)=181.65, p<0.0001$, for central; $F(3,40)=84.70, p<0.001$, for peripheral). Compared with the normal aged retinae (Figures $1(\mathrm{e})$ and $1(\mathrm{~m})$ ), the number of RGCs in central and peripheral regions of aged retinae was significant decreased since IOP of $45 \mathrm{mmHg}$ compared with the normal aged retinae (Figures 1(f), 1(h), 1(q), 1(n)-1(p), and 1(r)). However, significant loss of RGCs in central and peripheral retinae of young adult rats could not be detected until at $90 \mathrm{mmHg}$ (Figures 1(d), 1(q), 1(l), and 1(r)). Of note, RGC loss was more evident in aged retinae than young adult retinae at each IOP treatment (Figures 1(q) and 1(r)). These results were consistent with our previous data [9], indicating that aged retinae are more vulnerable to increased IOP compared to young adult retinae.

3.2. Microglia/Macrophages of Aged Retinae Were More Prone to Exhibit Proinflammatory Phenotype than That of Young Adult Retinae after High IOP Treatment. In glaucoma, activated microglia/macrophages could adopt proinflammatory phenotype that contributes to neuronal death by releasing neurotoxic factors [28]. These proinflammatory microglia/macrophages might upregulate CD68, a lysosomal marker indicative of phagocytic activity of microglia/macrophages, and antigen presentation molecule MHC-II [16]. Here, we detected the proinflammatory microglia/macrophages in the inner retina by double immunostaining of IBA1 and CD68/MHC-II (Figure 2). Two-way ANOVA analysis showed that aging significantly increases the number of $\mathrm{CD}^{+} 8^{+} \mathrm{IBA}^{+}$and $\mathrm{MHC}-\mathrm{II}^{+} \mathrm{IBA}^{+}$cells 3 days after IOP treatment $\left(F(1,40)=116.72, p<0.001\right.$, for $\mathrm{CD}^{+-}$ $\mathrm{IBA}^{+}$cells; $F(1,40)=156.89, p<0.001$, for $\mathrm{MHC}^{-\mathrm{II}^{+} \mathrm{IBA}^{+}}$ cells). IOP treatment also significantly increased the number of $\mathrm{CD} 68^{+} \mathrm{IBA}^{+}$and $\mathrm{MHC}-\mathrm{II}^{+} \mathrm{IBA}^{+}$cells $(F(3,40)=250.13$ , $p<0.001$, for $\mathrm{CD}^{+} 8^{+} \mathrm{IBA1}^{+}$cells; $F(3,40)=190.03, p<$ 0.001 , for $\mathrm{MHC}-\mathrm{II}^{+} \mathrm{IBA} 1^{+}$cells). The significant increases in $\mathrm{CD}^{+} 8^{+} \mathrm{IBA}^{+}$and $\mathrm{MHC}-\mathrm{II}^{+} \mathrm{IBA}^{+}$cells were detected in aged retinae since IOP of $60 \mathrm{mmHg}$ (Figures $2(\mathrm{~g}), 2(\mathrm{~h})$, $2(\mathrm{q}), 2(\mathrm{o}), 2(\mathrm{p})$, and $2(\mathrm{r}))$ and in young adult retinae at $90 \mathrm{mmHg}$ (Figures 2(d), 2(q), 2(l), and 2(r)). The number of $\mathrm{CD}_{68}{ }^{+} \mathrm{IBA}^{+}$and $\mathrm{MHC}-\mathrm{II}^{+} \mathrm{IBA}^{+}$cells in aged retinae was significantly higher than that of young adult retina at 60 and $90 \mathrm{mmHg}$ (Figures 2(q) and 2(r)). These findings suggested that increased IOP induces the proinflammatory activation of microglia/macrophages more easily in aged retinae than young adult retinae.

3.3. Aged Retinae Were More Prone to Induce Astrogliosis and C3 Deposition than Young Adult Retinae in Response to Increased IOP. We previously found that no significant difference of astrogliosis (quantified by the relative mean gray value of reactive astrocyte marker GFAP) exists between young adult and aged retinae under the same high IOP treatment [9]. In the present study, we assessed the astrogliosis represented as the relative mean gray value of 


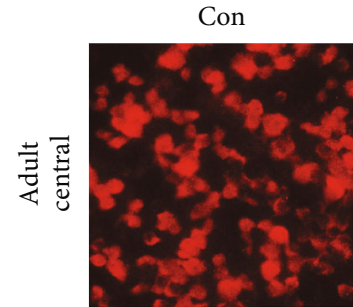

(a)

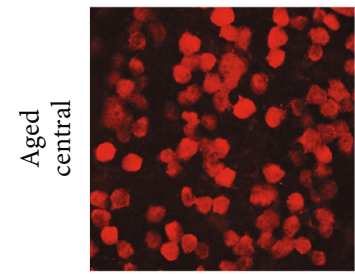

(e)

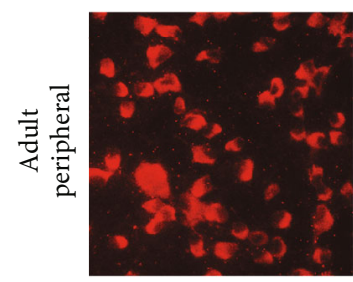

(i)

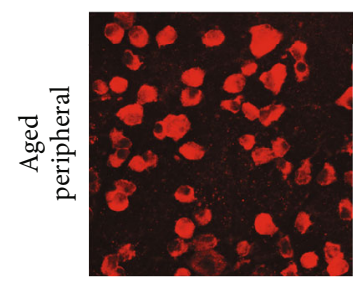

(m)
$45 \mathrm{~mm} \mathrm{Hg}$

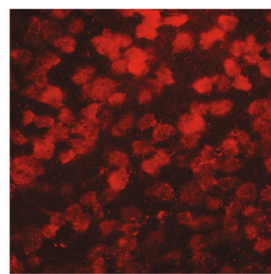

(b)

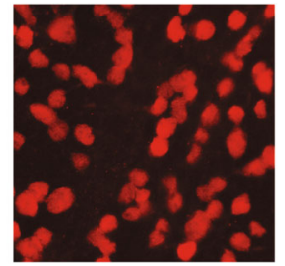

(f)

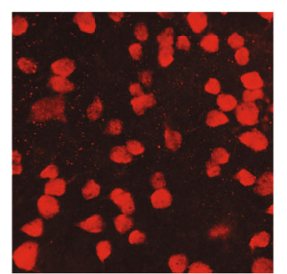

(j)

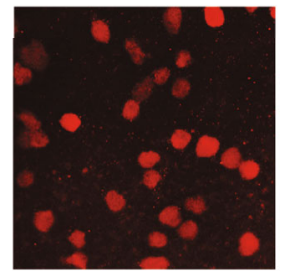

(n)

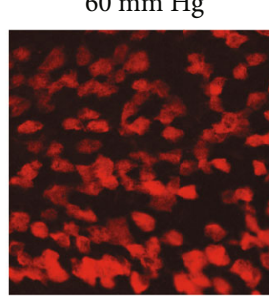

(c)

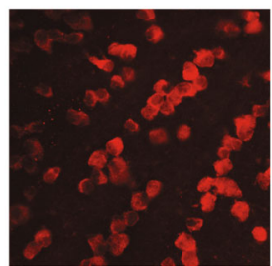

(g)

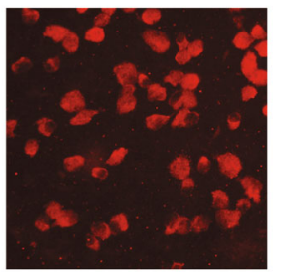

(k)

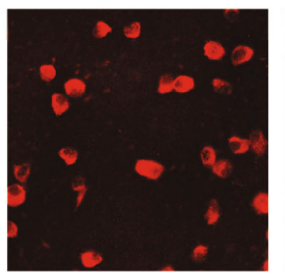

(o)
$90 \mathrm{~mm} \mathrm{Hg}$

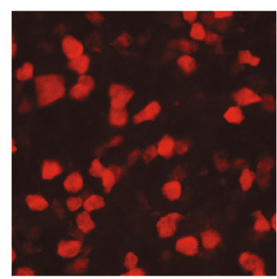

(d)

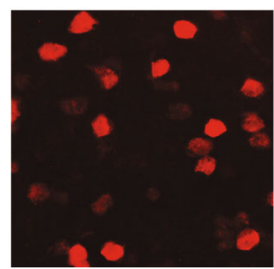

(h)

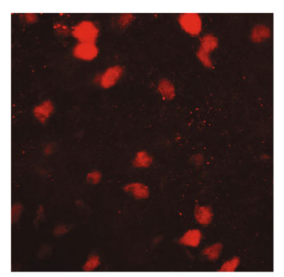

(l)

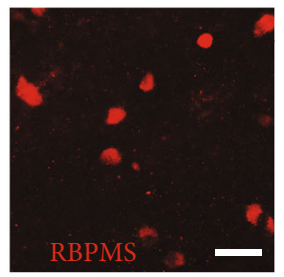

(p)

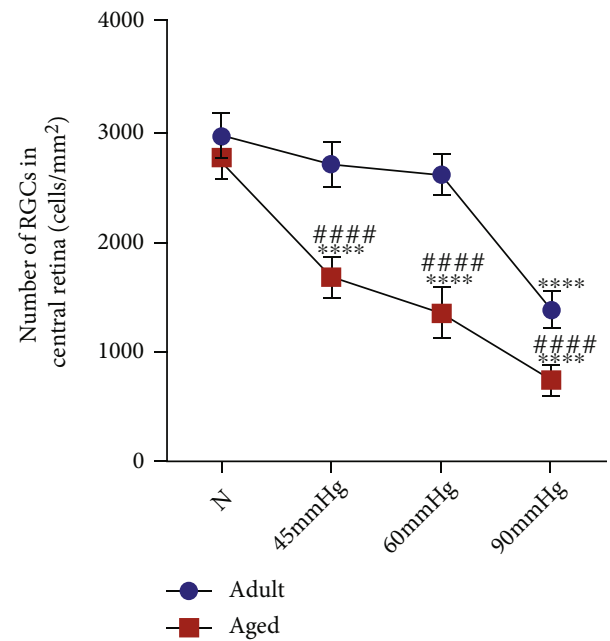

(q)

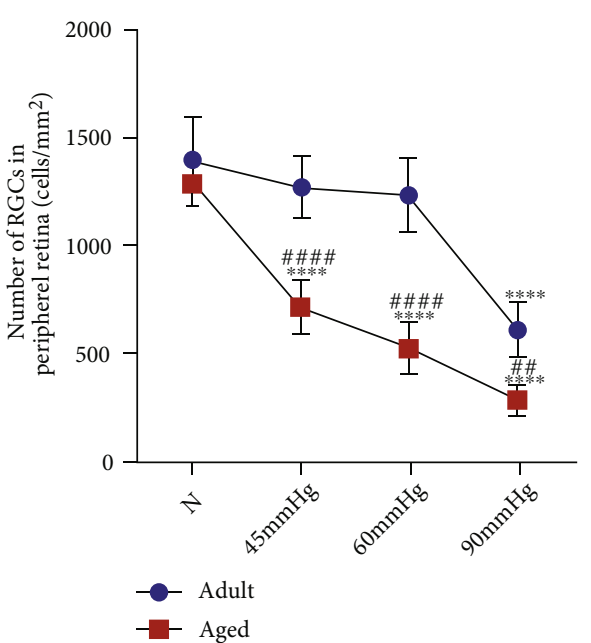

(r)

FIGURE 1: RGCs of aged retinae were more vulnerable to increased IOP than that of young adult retinae. Compared with the age-matched normal control (i, m), IOP treatment of $90 \mathrm{mmHg}$ was sufficient to induce significant loss of RGCs in central (d, q) and peripheral regions (l, r) of young adult retina 3 days after treatment. Since IOP of $45 \mathrm{mmHg}$, the aged retina showed significant loss of RGCs in central (f-h, q) and peripheral regions ( $\mathrm{n}-\mathrm{p}, \mathrm{r})$. RGC loss in aged retinae was significantly higher than that of young adult retinae at each IOP treatment ( $\mathrm{q}$, r) $\left({ }^{* * * *} p<0.0001\right.$ versus age-matched control; ${ }^{\# \#} p<0.01$, \#\#\#\# $p<0.0001$ versus matched part of young adult retina at the same IOP treatment. Scale bar: $100 \mu \mathrm{m})$. 
Con

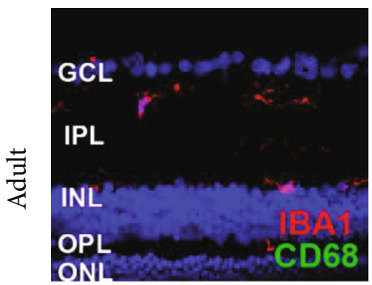

(a)

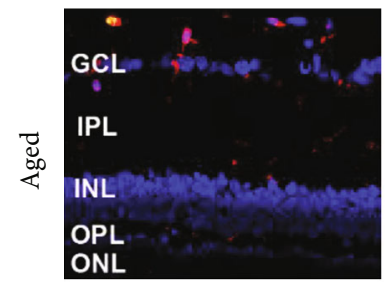

(e)

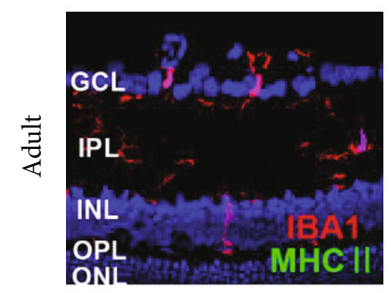

(m)
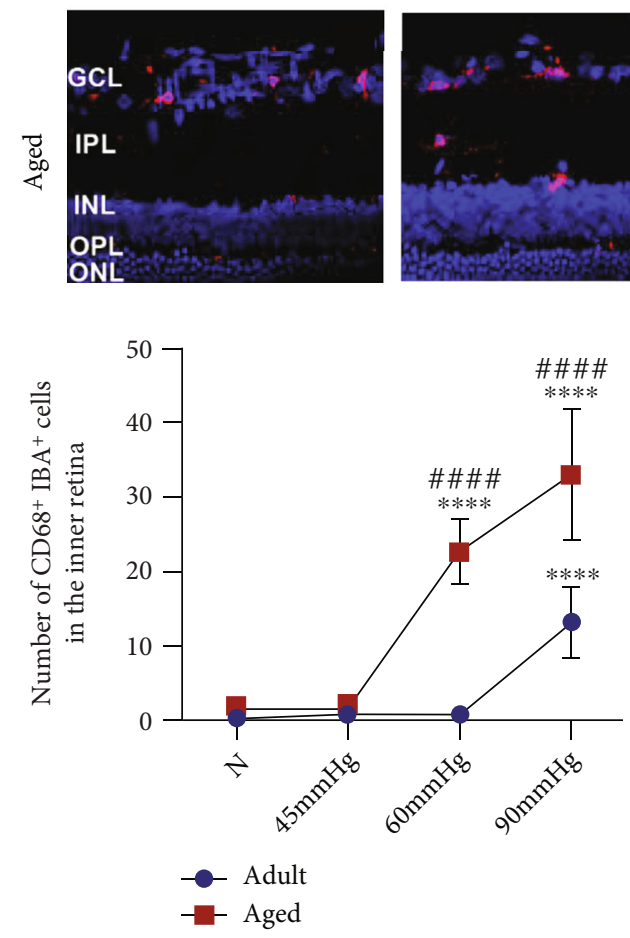

(q)
$45 \mathrm{~mm} \mathrm{Hg}$

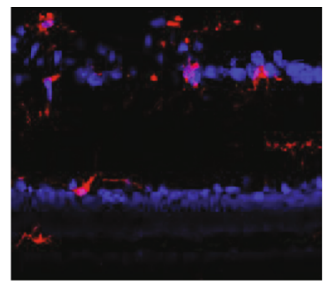

(b)

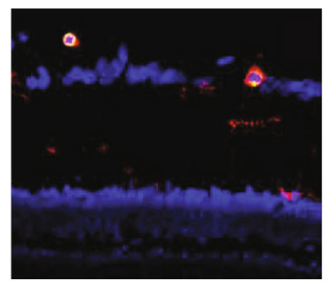

(f)

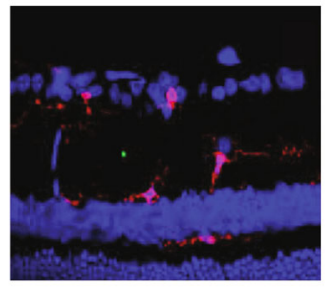

(n)
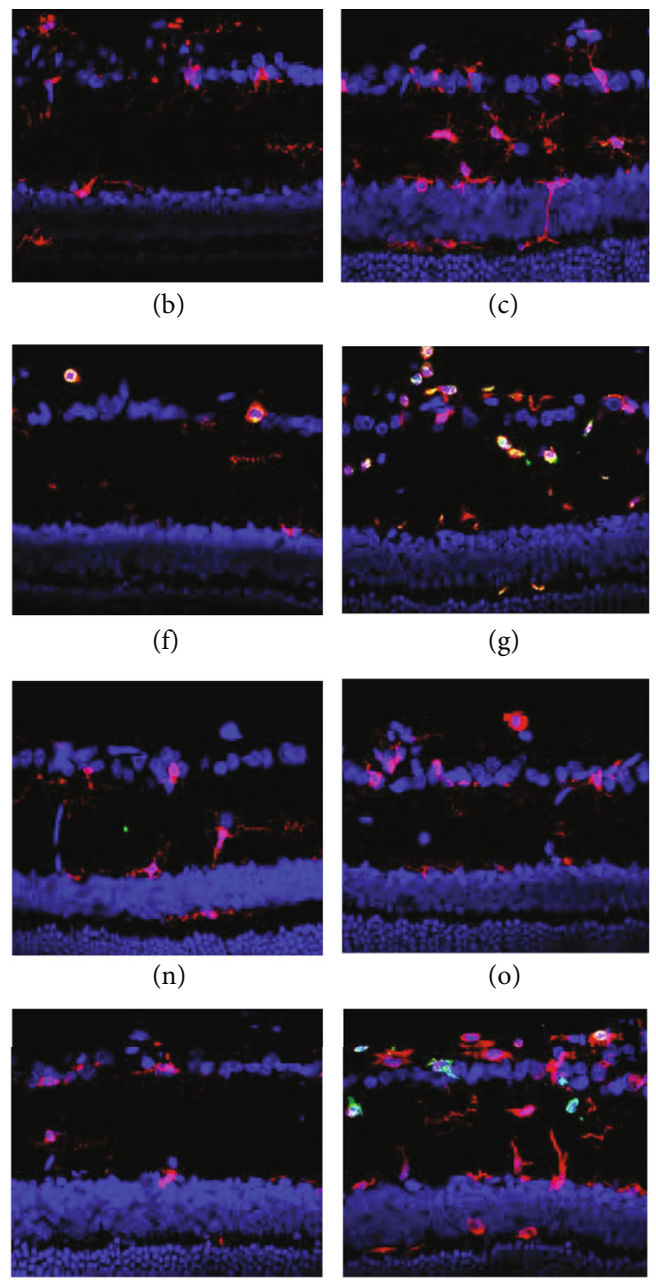

(c)

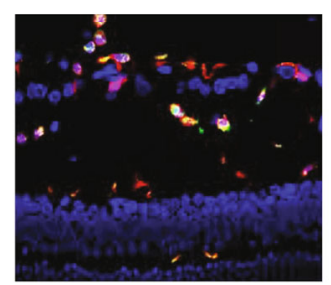

(g)

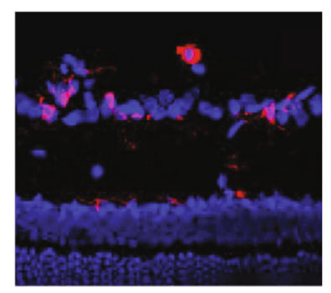

(o)
90 mm Hg

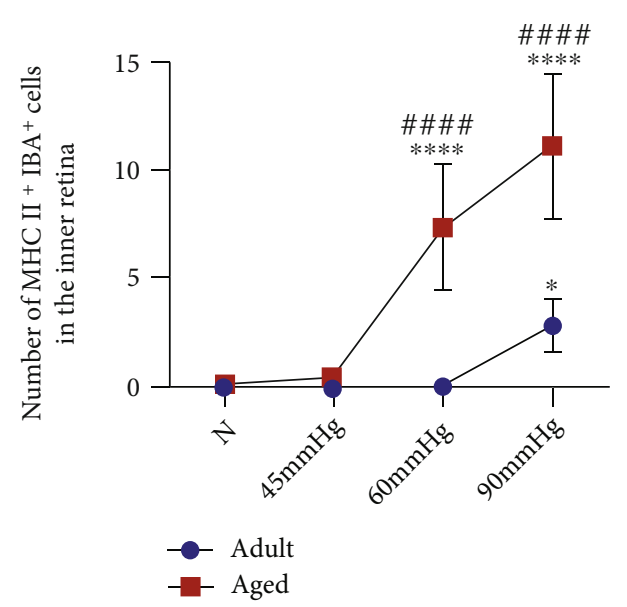

(r)

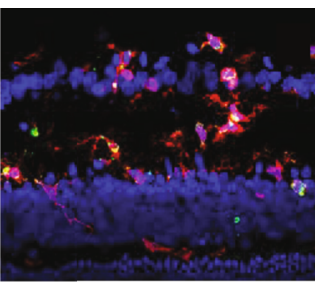

(d)

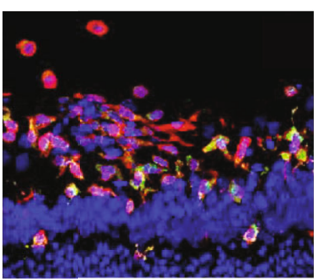

(h)

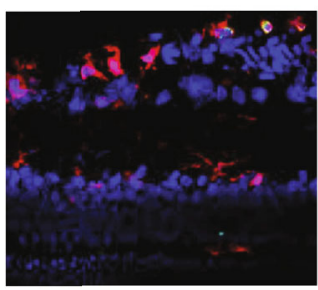

(p)

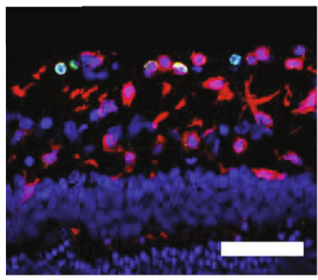

FIGURE 2: Microglia/macrophages were more prone to exhibit proinflammatory phenotype in aged retinae than that of young adult retinae after IOP treatment. Significant increase in $\mathrm{CD}_{6} 8^{+} \mathrm{IBAl}^{+}(\mathrm{d}, \mathrm{q})$ and $\mathrm{MHC}-\mathrm{II}^{+} \mathrm{IBA} 1^{+}$cells $(\mathrm{l}, \mathrm{r})$ was observed in young adult retinae at IOP of $90 \mathrm{mmHg}$. Aged retinae showed dramatic increase in $\mathrm{CD}_{68}^{+} \mathrm{IBAl}^{+}(\mathrm{g}-\mathrm{h}, \mathrm{q})$ and $\mathrm{MHC}^{-\mathrm{II}^{+} \mathrm{IBA1}} \mathrm{I}^{+}$cells $(\mathrm{o}-\mathrm{p}, \mathrm{r})$ since $\mathrm{IOP}$ of $60 \mathrm{mmHg}$. Compared with the young adult retinae, aged retinae induced more $\mathrm{CD}_{6} 8^{+} \mathrm{IBAl}^{+}$(q) and $\mathrm{MHC}^{-\mathrm{II}^{+} \mathrm{IBA1}}{ }^{+}$cells $(\mathrm{r})$ at 60 and $90 \mathrm{mmHg} 3$ days after IOP treatment (GCL: ganglion cell layer; IPL: inner plexiform layer; INL: inner nuclear layer; OPL: outer plexiform layer;

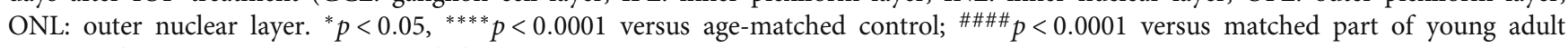
retina at the same IOP treatment. Scale bar: $50 \mu \mathrm{m}$ ). 
GFAP and relative $\mathrm{GFAP}^{+}$area in the inner retinae (Figure 3). Two-way ANOVA analysis showed that IOP treatment significantly increases the relative mean gray value of GFAP $(F(3,40)=39.64, p<0.0001)$. However, aging shows no effects on the relative mean gray value of GFAP in the inner retina after IOP treatment $(F(1,40)=0.13, p$ $=0.7213$ ) (Figure 3 ). Of note, we found that aging affects the positive staining area of GFAP after IOP treatment $(F(1,40)=13.61, p=0.0007)$. Moreover, IOP treatment significantly increased the $\mathrm{GFAP}^{+}$area in the inner retinae $(F(3,40)=27.18, p<0.0001)$. Significant increase in the $\mathrm{GFAP}^{+}$area in the young adult retinae was not detected until at IOP of $90 \mathrm{mmHg}$ (Figures 3(a)-3(d), YB). The GFAP ${ }^{+}$ area in aged retinae was increased since IOP of $60 \mathrm{mmHg}$ (Figures 3(e)-3(h), YB). Additionally, the $\mathrm{GFAP}^{+}$area in aged retinae was significantly higher than young adult retinae at $60 \mathrm{mmHg}$ (Figure 3, YB). These results suggested that strong astrogliosis is induced more easily in aged retinae than young adult retinae.

Reactive astrocytes might adopt neurotoxic phenotype in glaucoma to drive neurodegenerative process [29]. These neurotoxic astrocytes are marked by the production of complement C3 [30]. Recent studies identified that retinal deposition of C3 contributes to glaucomatous neurodegeneration [31]. We saw the upregulation of C3 within astrocytes in both aged and young adult retinae in response to elevated IOP (Figures 3(q) $-3(\mathrm{t})$ and $3(\mathrm{u})-3(\mathrm{x}))$. Further, we assessed the $\mathrm{C} 3$ deposition in the inner retinae by measurement of the relative mean gray value of $\mathrm{C} 3$ and $\mathrm{C}^{+}$areas (Figure 3, $\mathrm{ZA}$ and $\mathrm{ZB})$. We found that aging affects the relative mean gray value $(F(1,40)=11.05, p=0.0019)$ and positive staining area $(F(1,40)=28.24, p<0.0001)$ of C3. In addition, IOP treatment dramatically increased the relative mean gray value $(F(3,40)=47.58, \quad p<0.0001)$ and positive area $(F(3,40)=33.37, p<0.0001)$ of $\mathrm{C} 3$. We found that the relative mean gray value and positive area of C3 in aged retinae are not different from that of young adult retinae at the normal control (Figures 3(i) and 3(m), ZA and ZB). The increase in the relative mean gray value of $\mathrm{C} 3$ was detected in aged retinae since IOP of $45 \mathrm{mmHg}$, whereas the $\mathrm{C} 3^{+}$area was increased at 60 and $90 \mathrm{mmHg}$ (Figures 3(n)-3(p), ZA and $\mathrm{ZB})$. However, significant increase in the relative mean gray value and $\mathrm{C}^{+}$area could not be detected in young adult retinae until at IOP of $90 \mathrm{mmHg}$ (Figure 3(l), ZA and ZB). Moreover, aged retinae showed significantly higher levels of the relative mean gray value of the $\mathrm{C} 3$ at $60 \mathrm{mmHg}$ and $\mathrm{C}^{+}$area at 60 and $90 \mathrm{mmHg}$ than young adult retinae (Figure 3, ZA and $\mathrm{ZB}$ ). These results suggested that reactive astrocytes may be more primed to adopt neurotoxic phenotype in aged retinae than in young adult retinae, which is characterized by the higher levels of C3 deposition in the inner part of retinae.

3.4. High IOP Treatment Induced Higher Production of Proinflammatory Cytokines in Aged Retinae than in Young Adult Retinae. Proinflammatory microglia/macrophages and neurotoxic astrocytes produced various proinflammatory cytokines such as TNF and IL- $1 \beta$ to mediate neuroinflammatory response $[28,32]$. Here, we detected the protein levels of TNF and IL- $1 \beta$ in rat retinae by ELISA (Figures 4(a) and 4(b)). Two-way ANOVA results suggested that aging seriously affects the TNF and IL- $1 \beta$ protein levels after IOP treatment $(F(1,40)=320.48, p<0.0001$, for TNF; $F(1,40)=60.71, p<0.0001$, for IL-1 $\beta)$. Moreover, IOP treatment significantly increased the TNF and IL- $1 \beta$ protein levels $(F(3,40)=100.43, p<0.0001$, for TNF; $F(3,40)=$ $73.58, p<0.0001$, for IL-1 $\beta)$. We saw that TNF protein levels are dramatically increased in aged retinae since IOP of $45 \mathrm{mmHg}$ (Figure 4(a)). The increase in TNF protein levels in young adult retinae could not be detected until at IOP of $90 \mathrm{mmHg}$ (Figure 4(a)). The protein levels of IL-1 $\beta$ in aged retinae were significantly elevated since IOP of $60 \mathrm{mmHg}$ (Figure 4(b)). Young adult retinae showed modest but not statistically significant increase in IL- $1 \beta$ levels at $90 \mathrm{mmHg}(p=0.3323)$ (Figure $4(\mathrm{~b}))$. The protein levels of TNF in aged retinae were significantly higher than that of young adult retinae at each IOP treatment (Figure 4(a)). Aged retinae also showed higher degree of IL- $1 \beta$ protein levels than that of young adult retinae since IOP of $60 \mathrm{mmHg}$ (Figure 4(b)). Collectively, these data indicated that high IOP treatment induces stronger neuroinflammatory response in aged retinae compared to young adult retinae.

\section{Discussion}

The purpose of this study was to investigate if age of rats affects the retinal neuroinflammatory response in acute rat glaucoma. Here, we demonstrated that compared to young adult retinae, (1) RGCs in aged retinae are more susceptible to acute IOP elevation, (2) microglia/macrophages are more prone to adopt the proinflammatory phenotype in response to IOP elevation in aged retinae, (3) astrogliosis and C3 deposition are induced more easily in aged retinae, and (4) high IOP treatment induces higher levels of proinflammatory cytokines in aged retinae. These results supported that aged retinae induce more severe neuroinflammatory response than young adult retinae in acute glaucoma.

Loss of RGCs was the main pathological hallmark of glaucomatous degeneration [33]. In our previous study, loss of neurons at ganglion cell layer (GCL) after high IOP treatment was measured by counting the number of $\mathrm{NeuN}^{+}$cells in retinal cross-sections. Here, we quantified the loss of RGCs by counting RBPMS ${ }^{+}$(a specific marker of RGC in retina) cells in retinal whole mounts. The results of RGC loss in this study were consistent with the findings we have previously reported [9], which indicates the existence of agerelated susceptibility of RGC to elevated IOP.

In glaucoma, microglia/macrophages might transform into "ameboid" morphology and upregulate phagocytosisassociated protein CD68, antigen presentation molecule MHC-II, and other neurotoxic molecules, displaying the proinflammatory and phagocytic phenotypes $[34,35]$. In the present study, we saw the upregulation of CD68 and $\mathrm{MHC}-\mathrm{II}$ in $\mathrm{IBA} 1^{+}$microglia/macrophages of the retina after high IOP treatment. We found that significant increase in $\mathrm{CD}^{2} 8^{+} \mathrm{IBA}^{+}$and $\mathrm{MHC}-\mathrm{II}^{+} \mathrm{IBA}^{+}$cells could not be detected in young adult retinae until IOP of $90 \mathrm{mmHg}$. However, 

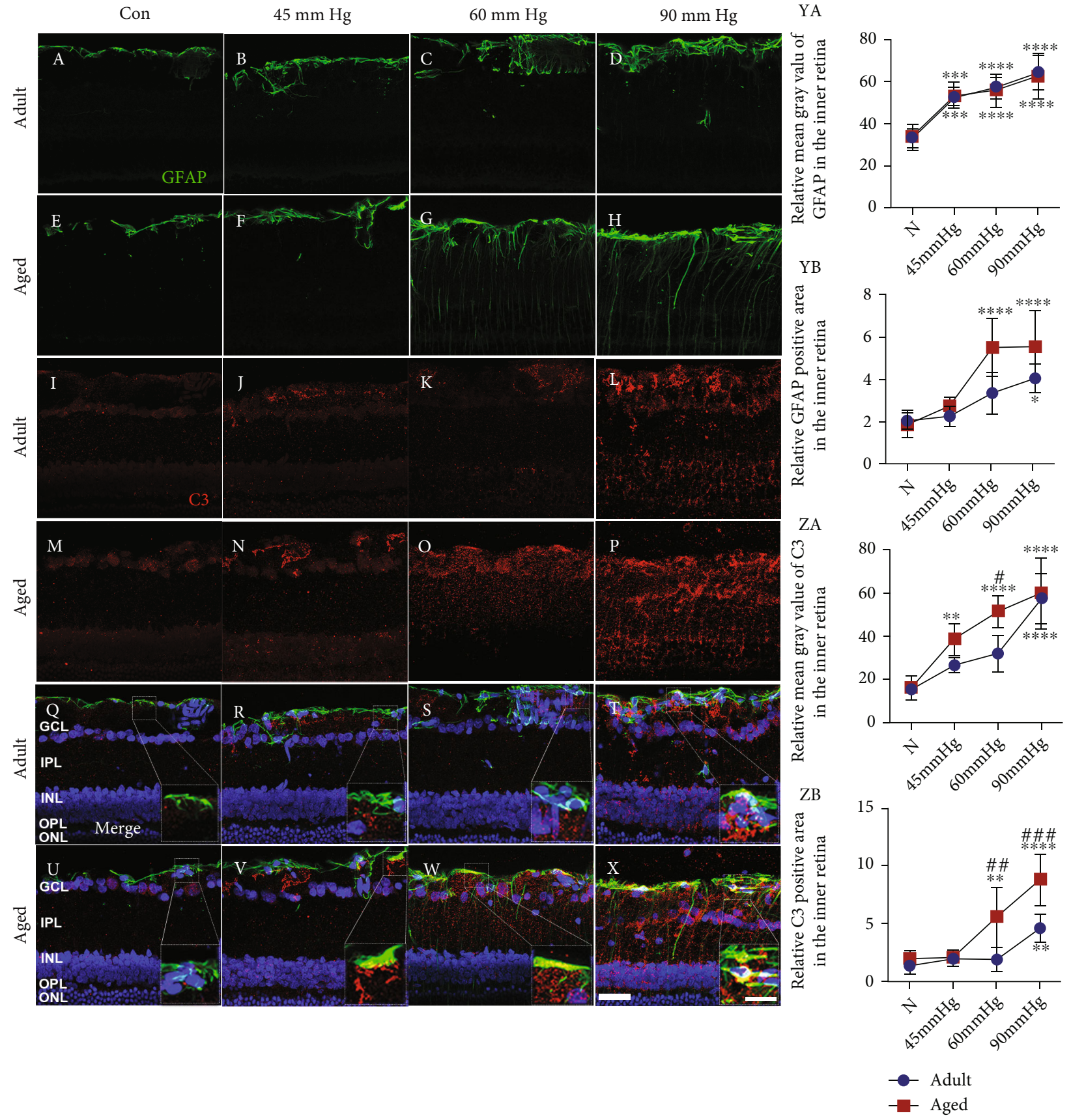

FIgURE 3: High IOP treatment induced astrogliosis and C3 deposition more easily in aged retinae than in young adult retinae. High IOP treatment induced the increase in GFAP and C3 staining in young adult (a-d, i-l) and aged retinae (e-h, m-p). Merged images showed colocalization (yellow) of C3 and GFAP staining, suggesting the formation of neurotoxic astrocytes in young adult (q- $\mathrm{t}$ ) and aged retinae $(\mathrm{u}-\mathrm{x}) 3$ days after treatment. Quantification of GFAP and C3 expression in the inner retina was presented as the relative mean gray value and relative positive area (YA-YB, ZA-ZB) (GCL: ganglion cell layer; IPL: inner plexiform layer; INL: inner nuclear layer; OPL: outer plexiform layer; ONL: outer nuclear layer. ${ }^{*} p<0.05,{ }^{* *} p<0.01,{ }^{* * *} p<0.001,{ }^{* * * *} p<0.0001$ versus age-matched control; ${ }^{\#} p<0.05$,

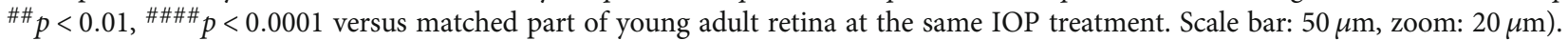

aged retinae increased $\mathrm{CD}^{+} 8^{+} \mathrm{IBA}^{+}$and $\mathrm{MHC}^{+} \mathrm{II}^{+} \mathrm{IBA}^{+}$ cells at $60 \mathrm{mmHg}$ and more as IOP increased. Moreover, the number of $\mathrm{CD}^{+} 8^{+} \mathrm{IBA}^{+}$and $\mathrm{MHC}-\mathrm{II}^{+} \mathrm{IBA}^{+}$cells in aged retinae was significantly higher than young adult retina at 60 and $90 \mathrm{mmHg}$. These findings indicated that microglia/ macrophages are more prone to adopt proinflammatory phenotype in aged retinae in response to elevated IOP.
Recent studies discovered several factors contribute to the age-related proinflammatory changes in retinal microglia, including the altered metabolism and reduced immunoregulatory signaling (e.g., CX3CL1-CX3CR1 and CD200CD200R signaling) from the retinal neurons [11]. In addition, Tang et al. unraveled that epigenetic modification may induce the aged-related proinflammatory alterations 


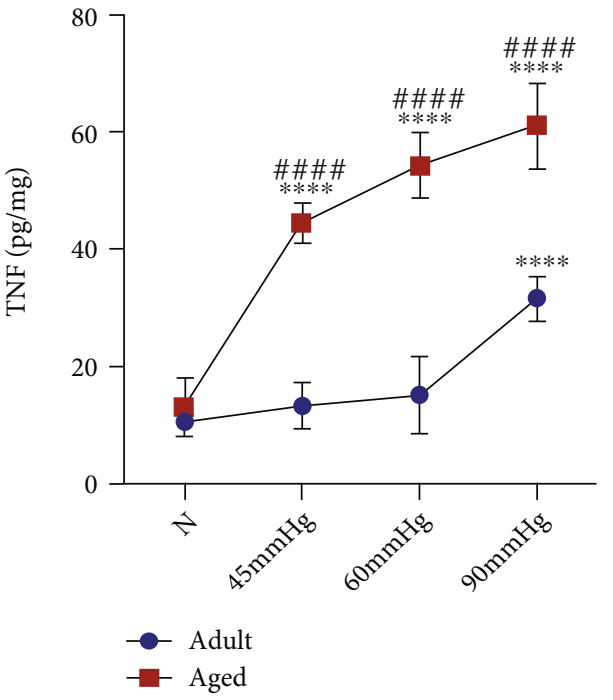

(a)

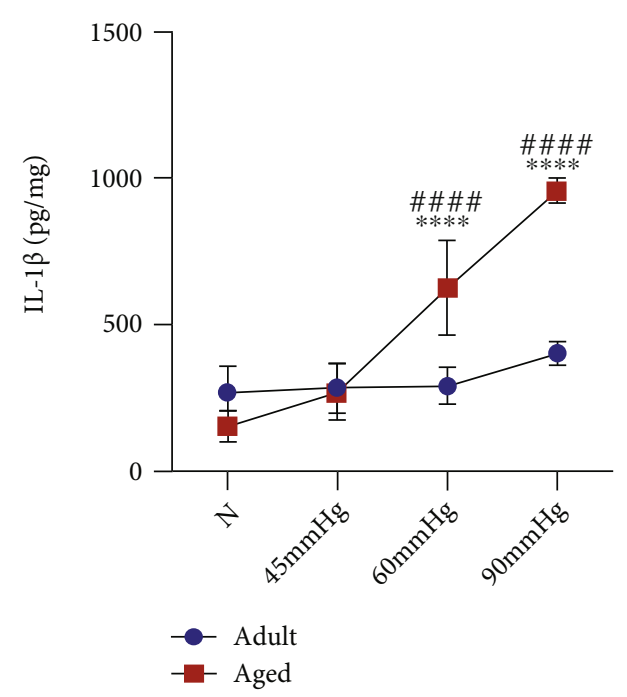

(b)

FIGURE 4: Aged retinae induced higher production of proinflammatory cytokines than young adult retinae after high IOP treatment. TNF levels were significantly increased in young adult retinae at IOP of $90 \mathrm{mmHg}$ and in aged retinae since IOP of $45 \mathrm{mmHg}$ (a). The protein levels of TNF in aged retinae were significantly higher than in young adult retinae at each IOP treatment (a). IL-1 $\beta$ levels were dramatically increased in aged retinae since IOP of $60 \mathrm{mmHg}$, while no significant increase in IL- $1 \beta$ was detected in young adult retinae after IOP treatment (b). IL-1 $\beta$ production in aged retinae was significantly higher than young adult retinae since IOP of $60 \mathrm{mmHg}(\mathrm{b})$ $\left({ }^{* * * *} p<0.0001\right.$ versus age-matched control; ${ }^{\# \# \#} p<0.0001$ versus matched part of young adult retina at the same IOP treatment).

in microglia [36]. In their reports, decreased expression of histone demethylases Jumonji domain-containing protein 3 (Jmjd3) and increased levels of Tri-methylation lysine 27 of histone H3 (H3K27me3) were detected in the midbrain of aged mice, which is accompanied by the upregulation of proinflammatory microglia markers (TNF, IL-6, and nitric oxide synthase 2 (NOS2)) and downregulation of antiinflammatory microglia marker Arginase-1 (Arg-1) [36].

Recent studies identified that neurotoxic astrocytes were major effectors to drive glaucomatous neurodegeneration $[29,37]$. However, the detailed mechanisms underlying how these neurotoxic astrocytes contribute to the damage of retinal neurons remained unclear. As the specific marker of neurotoxic astrocytes, complement $\mathrm{C} 3$ was tightly associated with neuroinflammation [30]. Lian et al. found that astrocyte-released C3 impaired dendritic morphology and network functions of cortical neurons through neuronal C3aR (C3a receptor) [38]. On the other hand, astrocytederived $\mathrm{C} 3$ could induce the proinflammatory activation of microglia via triggering the microglial C3aR [39]. In the present study, we firstly detected the degree of astrogliosis by immunostaining of reactive astrocyte marker GFAP. We found that high IOP treatment induces the increase in $\mathrm{GFAP}^{+}$area more easily in aged retinae than in young adult retinae. The obvious GFAP-positive processes in the inner plexiform layer were observed in aged retinae at IOP of $60 \mathrm{mmHg}$, while the $\mathrm{GFAP}^{+}$area in young adult retinae was limited to the ganglion cell layer under the same IOP. These findings implied that astrocytes in aged retinae may be more sensitive to increased IOP.

Next, we found upregulation of C3 within astrocytes in both young adult and aged retinae in response to increased
IOP, suggesting the formation of neurotoxic astrocytes. Recent studies have identified that C3 contributes to glaucomatous neurodegeneration [31]. Here, we showed higher degree of $\mathrm{C} 3$ deposition in aged retinae than in young adult retinae since IOP of $60 \mathrm{mmHg}$. These findings might provide evidence for the existence of age-related proinflammatory changes in retinal astrocytes. In fact, recent studies unraveled that normal aging can induce the proinflammatory changes of astrocytes in the mouse brain, which is characterized by the upregulation of genes involved in complement, inflammatory cytokines, and antigen presentation pathways [24]. Of note, it was identified that aging-induced proinflammatory changes of astrocytes is mediated by microgliareleased inflammatory signal (TNF, IL-1 $\beta$, and C1q) factors. This indicated that aging-induced alterations of microglia and astrocytes may be a coordinative process [24].

Proinflammatory microglia/macrophages and neurotoxic astrocytes were the main source of proinflammatory cytokines such as TNF and IL- $1 \beta$ in the context of glaucoma [40]. Several studies revealed that TNF/TNF receptor 1 (TNFR1) signaling can directly induce RGC death via proteolytic caspase cascade, mitochondrial dysfunction, and oxidative damage [41-43]. On the other hand, TNF and IL- $1 \beta$ could promote the proinflammatory activation of microglia/macrophages and astrocytes in the autocrine or amacrine manner, which further amplifies the neuroinflammatory response [44]. Thus, we detected the TNF and IL- $1 \beta$ protein levels to evaluate the degree of retinal neuroinflammatory response after different IOP treatments. We found that significant increase in TNF protein levels cannot be detected in young adult retinae until IOP of $90 \mathrm{mmHg}$. In addition, young adult retinae showed a slight but not 
statistically significant increase in IL- $1 \beta$ at $90 \mathrm{mmHg}$. However, aged retinae upregulate TNF and IL- $1 \beta$ levels at IOP of from 45 to $90 \mathrm{mmHg}$. Besides, upregulation of TNF and IL$1 \beta$ in aged retinae was significantly higher than that of young adult retina under the same IOP. These findings indicated that elevated IOP might induce a more severe neuroinflammatory response in aged retinae than young adult retinae.

Taken together, our findings highlighted that aging can seriously affect the degree of retinal neuroinflammatory response in acute rat glaucoma. This might explain the age-related vulnerability of RGCs to increased IOP. Further studies should identify the molecular mechanisms that drive the age-related proinflammatory changes within microglia/ macrophages and astrocytes, which may provide the promising target for glaucoma treatment.

\section{Data Availability}

The data used to support the findings of this study are available from the corresponding author upon request.

\section{Conflicts of Interest}

The authors declare no conflicts of interest.

\section{Acknowledgments}

This work was funded by the National Natural Science Foundation of China (No. 81900890, No. 81670858, and No. 81974134), Provincial Natural Science Foundation of Hunan, China (No. 2021JJ40991, No. 2021JJ70147), and China Postdoctoral Science Foundation (No. 2018M643004).

\section{References}

[1] H. Quigley and A. Broman, "The number of people with glaucoma worldwide in 2010 and 2020," The British Journal of Ophthalmology, vol. 90, no. 3, pp. 262-267, 2006.

[2] S. Syc-Mazurek and R. Libby, "Axon injury signaling and compartmentalized injury response in glaucoma," Progress in Retinal and Eye Research, vol. 73, article 100769, 2019.

[3] R. Casson, G. Chidlow, J. Wood, J. Crowston, and I. Goldberg, "Definition of glaucoma: clinical and experimental concepts," Clinical \& Experimental Ophthalmology, vol. 40, no. 4, pp. 341-349, 2012.

[4] R. Re, D. Messenio, G. Marano et al., "Monitoring the haemodynamic response to visual stimulation in glaucoma patients," Scientific Reports, vol. 11, no. 1, p. 13567, 2021.

[5] G. Guedes, J. C. Tsai, and N. A. Loewen, "Glaucoma and aging," Current Aging Science, vol. 4, no. 2, pp. 110-117, 2011.

[6] S. Philip, A. Najafi, A. Tantraworasin, L. Pasquale, and R. Ritch, "Nailfold capillaroscopy of resting peripheral blood flow in exfoliation glaucoma and primary open-angle glaucoma," JAMA Ophthalmology, vol. 137, no. 6, pp. 618-625, 2019.

[7] P. Dimitrov, B. Mukesh, C. McCarty, and H. Taylor, "Five-year incidence of bilateral cause-specific visual impairment in the
Melbourne Visual Impairment Project," Investigative Ophthalmology \& Visual Science, vol. 44, no. 12, pp. 5075-5081, 2003.

[8] K. Nouri-Mahdavi, D. Hoffman, A. L. Coleman et al., "Predictive factors for glaucomatous visual field progression in the Advanced Glaucoma Intervention Study," Ophthalmology, vol. 111, no. 9, pp. 1627-1635, 2004.

[9] C. Tan, T. Hu, M. C. Peng et al., “Age of rats seriously affects the degree of retinal damage induced by acute high intraocular pressure," Current Eye Research, vol. 40, no. 3, pp. 300-306, 2015.

[10] Y. Takihara, M. Inatani, K. Eto et al., "In vivo imaging of axonal transport of mitochondria in the diseased and aged mammalian CNS," Proceedings of the National Academy of Sciences of the United States of America, vol. 112, no. 33, pp. 1051510520, 2015.

[11] M. Chen, C. Luo, J. Zhao, G. Devarajan, and H. Xu, "Immune regulation in the aging retina," Progress in Retinal and Eye Research, vol. 69, pp. 159-172, 2019.

[12] H. Xu, M. Chen, and J. Forrester, "Para-inflammation in the aging retina," Progress in Retinal and Eye Research, vol. 28, no. 5, pp. 348-368, 2009.

[13] C. Baudouin, M. Kolko, S. Melik-Parsadaniantz, and E. Messmer, "Inflammation in glaucoma: from the back to the front of the eye, and beyond," Progress in Retinal and Eye Research, vol. 83, article 100916, 2021.

[14] M. Bariş and G. Tezel, "Immunomodulation as a neuroprotective strategy for glaucoma treatment," Current Ophthalmology Reports, vol. 7, no. 2, pp. 160-169, 2019.

[15] G. Tezel, "Molecular regulation of neuroinflammation in glaucoma: current knowledge and the ongoing search for new treatment targets," Progress in Retinal and Eye Research, no. article 100998, 2021.

[16] X. Wei, K. Cho, E. Thee, M. Jager, and D. Chen, "Neuroinflammation and microglia in glaucoma: time for a paradigm shift," Journal of Neuroscience Research, vol. 97, no. 1, pp. 70-76, 2019.

[17] C. Luo, X. Yang, A. Kain, D. Powell, M. Kuehn, and G. Tezel, "Glaucomatous tissue stress and the regulation of immune response through glial Toll-like receptor signaling," Investigative Ophthalmology \& Visual Science, vol. 51, no. 11, pp. 56975707, 2010.

[18] G. Howell, D. Macalinao, G. Sousa et al., "Molecular clustering identifies complement and endothelin induction as early events in a mouse model of glaucoma," The Journal of Clinical Investigation, vol. 121, no. 4, pp. 1429-1444, 2011.

[19] V. Haage, N. Elmadany, L. Roll et al., "Tenascin C regulates multiple microglial functions involving TLR4 signaling and HDAC1," Brain, Behavior, and Immunity, vol. 81, pp. 470483, 2019.

[20] N. Habib, C. McCabe, S. Medina et al., "Disease-associated astrocytes in Alzheimer's disease and aging," Nature Neuroscience, vol. 23, no. 6, pp. 701-706, 2020.

[21] S. Krasemann, C. Madore, R. Cialic et al., "The TREM2-APOE pathway drives the transcriptional phenotype of dysfunctional microglia in neurodegenerative diseases," Immunity, vol. 47, no. 3, pp. 566-81.e9, 2017.

[22] S. M. O’Neil, K. Witcher, D. McKim, and J. Godbout, "Forced turnover of aged microglia induces an intermediate phenotype but does not rebalance CNS environmental cues driving priming to immune challenge," Acta Neuropathologica Communications, vol. 6, no. 1, p. 129, 2018. 
[23] S. Patterson, "Immune dysregulation and cognitive vulnerability in the aging brain: Interactions of microglia, IL-1 $\beta$, BDNF and synaptic plasticity," Neuropharmacology, vol. 96, no. Part A, pp. 11-18, 2015.

[24] L. Clarke, S. Liddelow, C. Chakraborty, A. Münch, M. Heiman, and B. Barres, "Normal aging induces A1-like astrocyte reactivity," Proceedings of the National Academy of Sciences of the United States of America, vol. 115, no. 8, pp. E1896-E1905, 2018.

[25] C. Dan, T. Jian-Bin, W. Hui et al., "Synaptophysin expression in rat retina following acute high intraocular pressure," Acta Histochemica et Cytochemica, vol. 41, no. 6, pp. 173-178, 2008.

[26] A. Triviño, R. De Hoz, J. Salazar, A. Ramírez, B. Rojas, and J. Ramírez, "Distribution and organization of the nerve fiber and ganglion cells of the human choroid," Anatomy and Embryology, vol. 205, pp. 417-430, 2002.

[27] T. Hu, S. Wang, L. Zeng, K. Xiong, D. Chen, and J. Huang, "Regional expression of act-MMP3 contributes to the selective loss of neurons in ganglion cell layers following acute retinal ischemia/reperfusion injury," Current Eye Research, vol. 45, no. 5, pp. 591-603, 2020.

[28] M. Madeira, R. Boia, P. Santos, A. Ambrósio, and A. Santiago, "Contribution of microglia-mediated neuroinflammation to retinal degenerative diseases," Mediators of Inflammation, vol. 2015, Article ID 673090, 15 pages, 2015.

[29] K. Guttenplan, B. Stafford, R. N. el-Danaf et al., "Neurotoxic reactive astrocytes drive neuronal death after retinal injury," Cell Reports, vol. 31, no. 12, article 107776, 2020.

[30] S. Liddelow, K. Guttenplan, L. Clarke et al., "Neurotoxic reactive astrocytes are induced by activated microglia," Nature, vol. 541, no. 7638, pp. 481-487, 2017.

[31] A. Bosco, S. Anderson, K. Breen et al., "Complement C3targeted gene therapy restricts onset and progression of neurodegeneration in chronic mouse glaucoma," Molecular Therapy, vol. 26, no. 10, pp. 2379-2396, 2018.

[32] G. Tezel, X. Yang, C. Luo, J. Cai, and D. Powell, “An astrocytespecific proteomic approach to inflammatory responses in experimental rat glaucoma," Investigative Ophthalmology \& Visual Science, vol. 53, no. 7, pp. 4220-4233, 2012.

[33] Y. Abdul, N. Akhter, and S. Husain, "Delta-opioid agonist SNC-121 protects retinal ganglion cell function in a chronic ocular hypertensive rat model," Investigative Ophthalmology \& Visual Science, vol. 54, no. 3, pp. 1816-1828, 2013.

[34] F. Walker, M. Nilsson, and K. Jones, "Acute and chronic stress-induced disturbances of microglial plasticity, phenotype and function," Current Drug Targets, vol. 14, no. 11, pp. 12621276, 2013.

[35] B. Rojas, B. Gallego, A. Ramírez et al., "Microglia in mouse retina contralateral to experimental glaucoma exhibit multiple signs of activation in all retinal layers," Journal of Neuroinflammation, vol. 11, no. 1, p. 133, 2014.

[36] Y. Tang, T. Li, J. Li et al., "Jmjd3 is essential for the epigenetic modulation of microglia phenotypes in the immune pathogenesis of Parkinson's disease," Cell Death and Differentiation, vol. 21, no. 3, pp. 369-380, 2014.

[37] J. Sterling, M. Adetunji, S. Guttha et al., "GLP-1 receptor agonist NLY01 reduces retinal inflammation and neuron death secondary to ocular hypertension," Cell Reports, vol. 33, no. 5, article 108271, 2020.

[38] H. Lian, L. Yang, A. Cole et al., "NF $\kappa B$-Activated Astroglial Release of Complement C3 Compromises Neuronal Morphol- ogy and Function Associated with Alzheimer's Disease," Neuron, vol. 85, no. 1, pp. 101-115, 2015.

[39] A. Litvinchuk, Y. Wan, D. Swartzlander et al., "Complement C3aR Inactivation Attenuates Tau Pathology and Reverses an Immune Network Deregulated in Tauopathy Models and Alzheimer's Disease," Neuron, vol. 100, no. 6, pp. 1337-1353, 2018.

[40] G. Tezel, L. Li, R. Patil, and M. Wax, “TNF-alpha and TNFalpha receptor-1 in the retina of normal and glaucomatous eyes," Investigative Ophthalmology \& Visual Science, vol. 42, no. 8, pp. 1787-1794, 2001.

[41] G. Tezel and M. Wax, "Increased production of tumor necrosis factor-alpha by glial cells exposed to simulated ischemia or elevated hydrostatic pressure induces apoptosis in cocultured retinal ganglion cells," The Journal of Neuroscience, vol. 20, no. 23, pp. 8693-8700, 2000.

[42] G. Tezel and X. Yang, "Caspase-independent component of retinal ganglion cell death, in vitro," Investigative Ophthalmology \& Visual Science, vol. 45, no. 11, pp. 4049-4059, 2004.

[43] X. Yang, C. Luo, J. Cai et al., "Neurodegenerative and inflammatory pathway components linked to TNF- $\alpha$ /TNFR1 signaling in the glaucomatous human retina," Investigative Ophthalmology \& Visual Science, vol. 52, no. 11, pp. 8442 8454, 2011.

[44] L. Han, D. Zhang, T. Tao et al., "The role of N-glycan modification of TNFR1 in inflammatory microglia activation," Glycoconjugate Journal, vol. 32, no. 9, pp. 685-693, 2015. 